\title{
Abstract
}

\section{Cascade of HIV Prevention, Care, and Treatment Services in Morocco in 2019}

Noureddine Sakhri, MD, MPH

Ministry of Health, Rabat, Morocco

Corresponding Author:

Noureddine Sakhri, MD, MPH

Ministry of Health

71, Ibn Sina Agdal

Rabat, 10000

Morocco

Phone: 212662751704

Email: $\underline{\text { sakhriph@gmail.com }}$

\section{Abstract}

Background: The HIV care cascade is a way to show the proportion of people living with HIV (PLHIV) engaged at each stage of HIV care. Analyzing an HIV testing, care, and treatment cascade provides a framework for evaluating and improving service delivery.

Objective: The objective of this study is to analyze the continuums of HIV prevention, care, and treatment services at the national level in Morocco for the period 2015-2019.

Methods: This is a retrospective study concerning the reference centers for HIV care in Morocco carried out in 2019. Three types of cascades of HIV prevention and care were studied: a transversal cascade at the national level (2019), a longitudinal cascade for newly diagnosed PLHIV (between 2015 and 2017), and a cascade of prevention of mother-to-child transmission of HIV (PMTCT) among pregnant women (2016-2017). The study process included collecting the data needed to construct the cascade and determining the magnitude of the deviations at each stage of the cascade.

Results: For the transversal cascade of the year 2019, the objectives of the three 90s were achieved except for the 1 st 90 with a difference of $12 \%$. For the longitudinal cascade after the start of treatment between 2015-2017, retention under treatment at 48 months was $83.3 \%$, at 36 months was $83.8 \%$, and at 24 months $91.0 \%$. The 48 -month loss to follow-up rate was $12.5 \% ; 7.0 \%$ at 36 months; and $4.0 \%$ at 24 months. More than 90\% of PLHIV started antiretrovirals within 3 months of diagnosis. The 2016-2017 PMTCT cascade conducted on 13 pregnant women according to available data indicated the absence of transmission of HIV from mothers to their children.

Conclusions: Our 2019 HIV cascade study demonstrated several successes. The achievement of the three 90s except for the first objective (of people who know their status), good retention of PLHIV in long-term treatment, and the success of PMTCT especially since Morocco aims to validate the elimination of mother-to-child transmission.

(iproc 2022;8(1):e36373) doi: $\underline{10.2196 / 36373}$

\section{KEYWORDS}

Cascade; HIV; mother-to-child transmission of HIV; PMTCT; proportion of people living with HIV; PLHIV

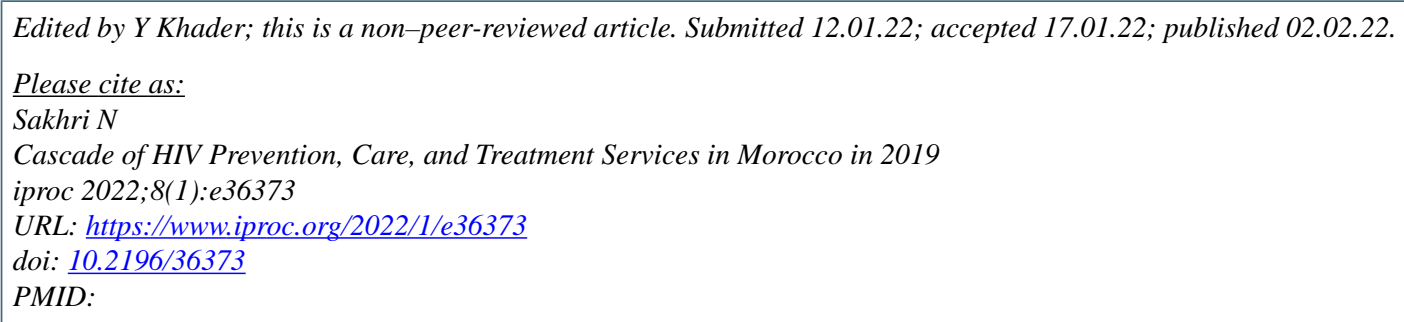


CNoureddine Sakhri. Originally published in Iproceedings (https://www.iproc.org), 02.02.2022. This is an open-access article distributed under the terms of the Creative Commons Attribution License (https://creativecommons.org/licenses/by/4.0/), which permits unrestricted use, distribution, and reproduction in any medium, provided the original work, first published in Iproceedings, is properly cited. The complete bibliographic information, a link to the original publication on https://www.iproc.org/, as well as this copyright and license information must be included. 